\title{
Concept and set-up of an IR-gas sensor construction kit
}

Ingo Sieber, Gernot Perner, Ulrich Gengenbach

Ingo Sieber, Gernot Perner, Ulrich Gengenbach, "Concept and set-up of an IR-gas sensor construction kit," Proc. SPIE 9793, Education and Training in Optics and Photonics: ETOP 2015, 97931E (8 October 2015); doi: $10.1117 / 12.2223110$

Event: Education and Training in Optics and Photonics: ETOP 2015, 2015, Bordeaux, France 


\title{
Concept and set-up of an IR-gas sensor construction kit
}

\author{
Ingo Sieber, Gernot Perner, Ulrich Gengenbach \\ Karlsruhe Institute of Technology, Institute for Applied Computer Science, Hermann-von- \\ Helmholtz-Platz 1, 76344 Eggenstein-Leopoldshafen, Germany
}

\begin{abstract}
The paper presents an approach to a cost-efficient modularly built non-dispersive optical IR-gas sensor (NDIR) based on a construction kit. The modularity of the approach offers several advantages: First of all it allows for an adaptation of the performance of the gas sensor to individual specifications by choosing the suitable modular components. The sensitivity of the sensor e.g. can be altered by selecting a source which emits a favorable wavelength spectrum with respect to the absorption spectrum of the gas to be measured or by tuning the measuring distance (ray path inside the medium to be measured). Furthermore the developed approach is very well suited to be used in teaching. Together with students a construction kit on basis of an optical free space system was developed and partly implemented to be further used as a teaching and training aid for bachelor and master students at our institute. The components of the construction kit are interchangeable and freely fixable on a base plate. The components are classified into five groups: Sources, reflectors, detectors, gas feed, and analysis cell.
\end{abstract}

Source, detector, and the positions of the components are fundamental to experiment and test different configurations and beam paths. The reflectors are implemented by an aluminum coated adhesive foil, mounted onto a support structure fabricated by additive manufacturing. This approach allows derivation of the reflecting surface geometry from the optical design tool and generating the 3D-printing files by applying related design rules. The rapid fabrication process and the adjustment of the modules on the base plate allow rapid, almost LEGOß-like, experimental assessment of design ideas.

Subject of this paper is modeling, design, and optimization of the reflective optical components, as well as of the optical subsystem. The realization of a sample set-up used as a teaching aid and the optical measurement of the beam path in comparison to the simulation results are shown as well.

Keywords: IR-gas sensor, construction kit, optical modeling and simulation, design optimization

\section{INTRODUCTION}

IR gas sensors are used in different fields, amongst others in safety engineering or in the field of quality control. Hence, a huge demand for inexpensive gas sensors exists. Operation of IR analyzers is based on the absorption of certain wavelengths of infrared radiation specific to individual gases. In the wavelength range of 3.0-5.2 $\mu \mathrm{m}$, the characteristic absorption bands of many gases and vapors allow to set up measuring systems combining an IR source, an absorption cell, a wavelength filter (or spectrometer, respectively), and a detector element (Fig. 1).

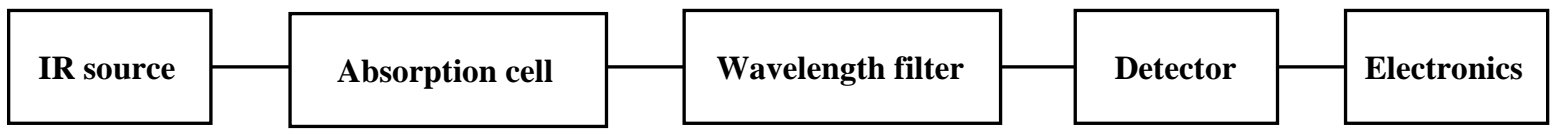

Figure 1: Schematic representation of an IR gas sensor [1].

Quantified IR gas analysis employs the Lambert-Beer law which relates the measured intensity $\mathrm{I}_{\lambda}$ with the reference intensity $\mathrm{I}_{0 \lambda}$ (see Eq.1) (e.g. [2], [3]):

(Eq. 1)

$$
\begin{array}{ll}
\log \frac{I \lambda}{I 0 \lambda} & =-\varepsilon \cdot c \cdot d=-E \text { with: } \\
E & =\text { extinction } \\
\varepsilon & =\text { extinction coefficient } \\
c & =\text { concentration of substance } \\
d & =\text { beam path in the substance }
\end{array}
$$

On the basis of the Lambert Beer law, any component in a gas mix can be determined quantitatively from its IR spectrum.

Education and Training in Optics and Photonics: ETOP 2015, edited by Eric Cormier, Laurent Sarger Proc. of SPIE Vol. 9793, 97931E · C 2015 SPIE, IEEE, OSA, ICO · doi: 10.1117/12.2223110 
NDIR are relatively simple in their construction and are suited for single gas detection. In their most simple setup, the analysis chambers are linear arrays in which the IR emitter and the IR detectors are situated at opposite ends. However, the linear, mostly relatively short ray path is a major constraint in the practical application of these sensors, as the gases to be detected must have a certain minimum absorption. To detect even weakly absorbing gases, the optical beam path in the analysis chamber therefore needs to be extended which can be achieved by convoluting the beam path and adding additional reflecting elements [4]. The integration of beamfocusing elements is becoming increasingly important in respect to achieve sufficient loading of the detector.

This paper is organized as follows: At first, the concept of the IR-sensor construction kit is presented. The following section deals with the modeling and design of the reflective components and the subsystem of the sample set-up. Section four is about the constructive realization of the construction kit's elements and the realization of the sample set-up of the optical subsystem as well as about optical measurements of the beam path. The paper is closed with the conclusions.

\section{CONCEPT OF THE IR-SENSOR CONSTRUCTION KIT}

The concept of the construction kit to be applied as teaching aid is based on the usage of different functional components which have to be freely arrangeable on a base plate to offer the functionality of a tunable NDIR (see Fig. 2).

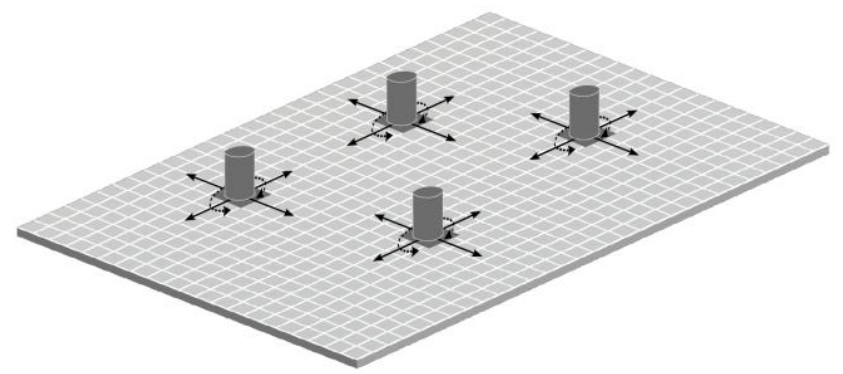

Figure 2. Concept of the base plate and the arrangement of the individual components.

A prerequisite of the concept of the construction kit is the modularity of the different components which can be classified into five groups [5]:

- Sources,

- reflectors,

- detectors,

- modular gas feed, and

- reconfigurable analysis cell.

The concept provides an exchangeability of the components source and detector, and the appropriate reflectors. Combined with the reconfigurable analysis cell this concept allows to build up an IR-gas sensor tailored to measuring a particular gas with its specific absorption band with a predefined sensitivity. The reconfigurability of the analysis cell enables in combination with appropriate reflectors the tuning of the path length of the beam in the medium to be detected.

\section{Optical modules}

To ensure the modularity of the optical components specific mounting structures were designed in a way to enable an assembly with different components of their group to form an optical module. Fig. 3 shows the models of the optical modules for the component groups sources, detectors, and reflectors. All components have to be shiftable in the plane of the base plate. Since the optical plane is defined by the position of the vertex of the reflector the mounting structure of the source as well as that of the detector have to be adjustable in their height. The starting direction of the beam is defined by the source module, so only the mounting structures of the reflector and the detector module have to be rotatable about the axis perpendicular to the base plate. 

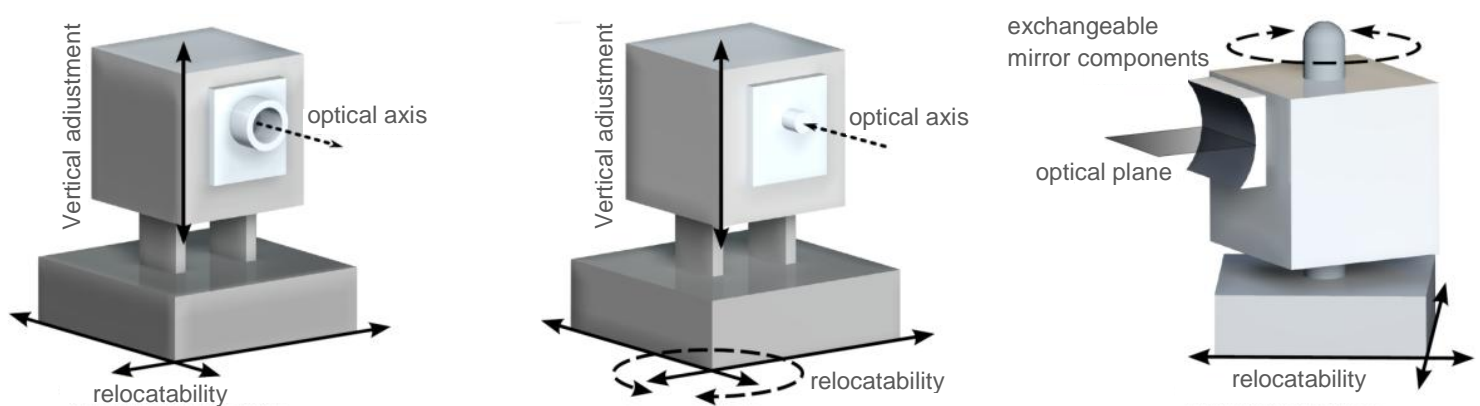

Figure 3. Models of the optical modules of the component groups sources, detectors, and reflectors (from left to right).

In the sample set-up realization of the reflection coating was carried out by means of an adhesive aluminum coated foil with a measured reflectivity of $86.4 \%$ in the wavelength range around $4.2 \mu \mathrm{m}$. This approach limits the reflector shape to $2.5 \mathrm{D}$. The spatial beam forming of the irradiance has to be carried out by two of the $2.5 \mathrm{D}$ reflectors: one of which is responsible for the beam forming perpendicular to the base plate, the other for beam forming in the plane of the base plate.

\section{MODELING AND DESIGN OF AN OPTICAL SAMPLE SET-UP}

The optical sample set-up to be modeled and designed consists of modules of the three groups sources, detectors, and reflectors. Neither the analysis cell nor the gas feed will be considered in this design. The optical concept followed in designing the sample set-up provides four mirrors: two mirrors for beam collimation of the source irradiance and two mirrors for beam focusing on the detector (see Fig. 4). To be able to easily tune the path length of the beam path the source and its two collimating mirrors as well as the detector together with its focusing mirrors will be arranged to form an independently moveable unit (see Fig. 4).

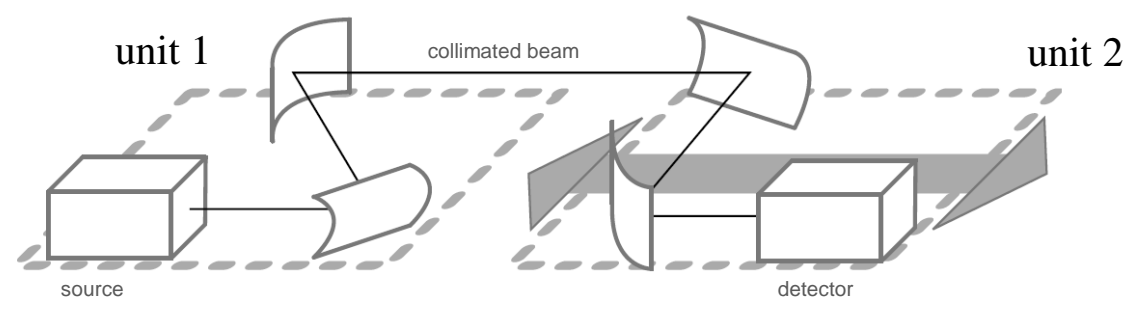

Figure 4. Schematic of the design of the optical subsystem using two 2.5D-shaped mirrors for beam collimation and beam focusing, respectively.

In the specific set-up source and detector components are chosen to meet the absorption spectrum of carbon dioxide $\left(\mathrm{CO}_{2}\right)$ while ideally the sensitivity of the sensor will be adjusted by the path length of the beam path in the medium, in the sample set-up specified by the arrangement and the design of the reflectors.

As a source of the sample set-up the broadband IR source IR-55 including reflector was chosen [6]. The irradiance characteristic of this source is shown in Fig. 5.

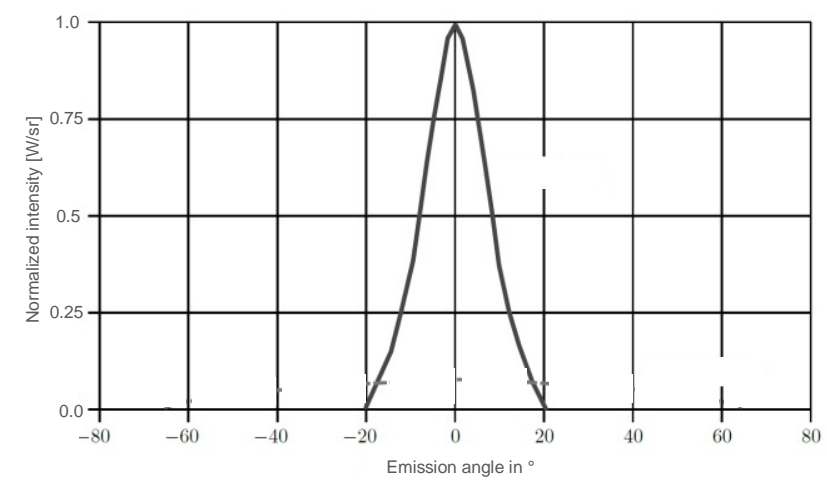

Figure 5. Irradiance characteristic of the IR-source IR-55 [6]. 
For detection of the transmitted irradiance the thermopile IR-Detector TS1 $x 200 B-B-D 2.4-1-K r-\mathrm{CO}_{2}$ [7] is used. Since a thermopile detector is a broad band detector a filter was used to cut out the relevant spectrum around the peak of the $\mathrm{CO}_{2}$ absorption band centered at around $4.2 \mu \mathrm{m}$. The principle absorption band of $\mathrm{CO}_{2}$ and the filter characteristic are shown in Fig. 6.
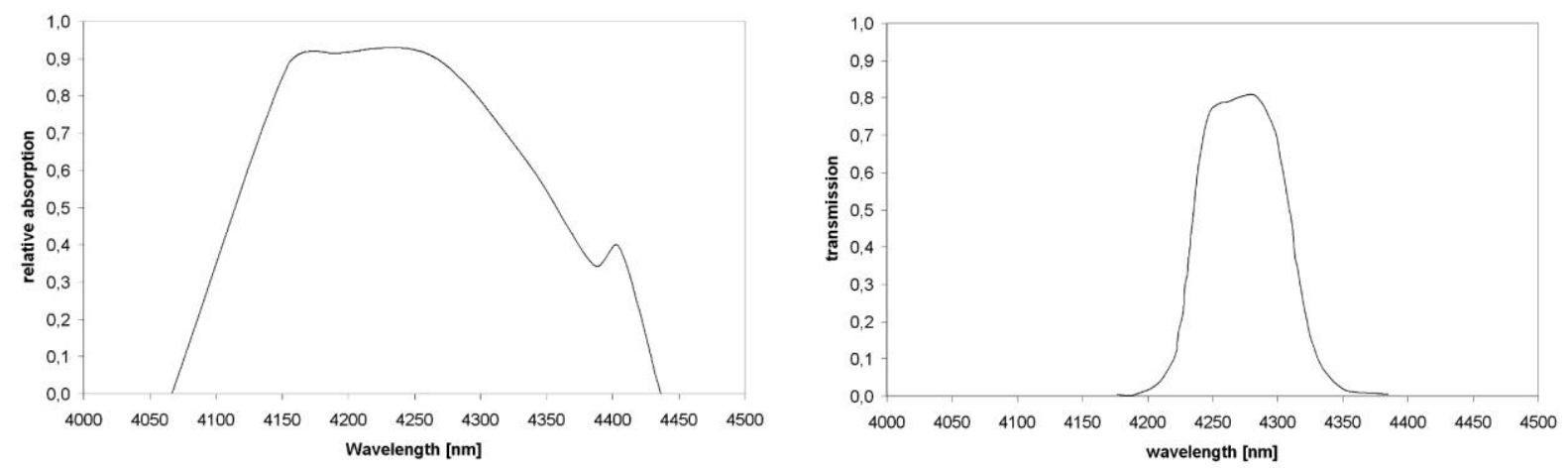

Figure 6. left: Principle absorption band of $\mathrm{CO} 2$; right: transmission characteristic of the filter used [5].

With these requirements the mirrors as well as the design of the subsystem were optimized. Fig. 7 shows the top view of the optimized subsystem model including some calculated beam paths. Shown are the components source, mirror 1 to 4 , and the detector. The mounting and alignment structures of the mirrors are depicted by circles, the mountings of source and detector by rectangles. Mirrors 1 and 3 are curved along the x-axis performing a beam modification in the direction perpendicular to the base plate while mirrors 2 and 4 have a curvature along the y-axis, hence performing a beam modification in the plane of the base plate.

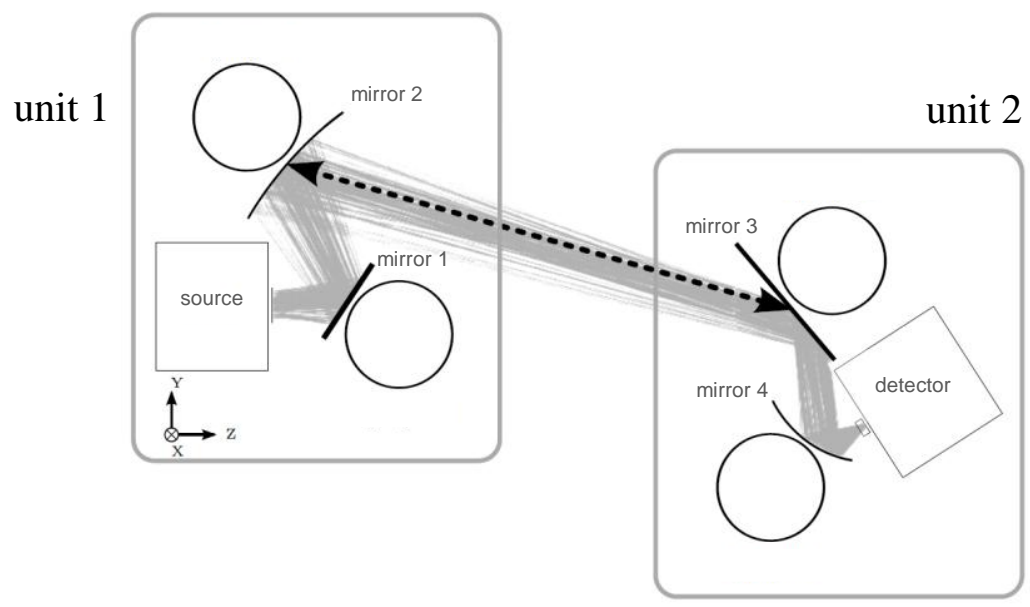

Figure 7. Top view of the optimized subsystem model including calculated beam paths.

Based on the optimized model of the optical subsystem the optical modules were constructed, the sample set-up was realized, and measurements of the beam path were conducted.

\section{SET-UP OF THE OPTICAL SUBSYSTEM AND OPTICAL MEASUREMENTS}

Fig. 8 shows the constructed modules of the IR-gas sensor construction kit which will be used as a teaching aid at our institute. The choice of source, detector types, and positions of the modules are fundamental to experiment and allows testing of different configurations and beam paths. In case of the sample set-up the reflecting surfaces are realized by an aluminum coated adhesive foil, which is mounted onto a mounting structure fabricated by additive manufacturing. This approach allows derivation of the reflecting surface geometry from the optical design tool and generating the 3D-printing files by applying design rules related to the adhesive foil and the additive manufacturing process. This rapid fabrication process allows quick experimental assessment of design ideas. 

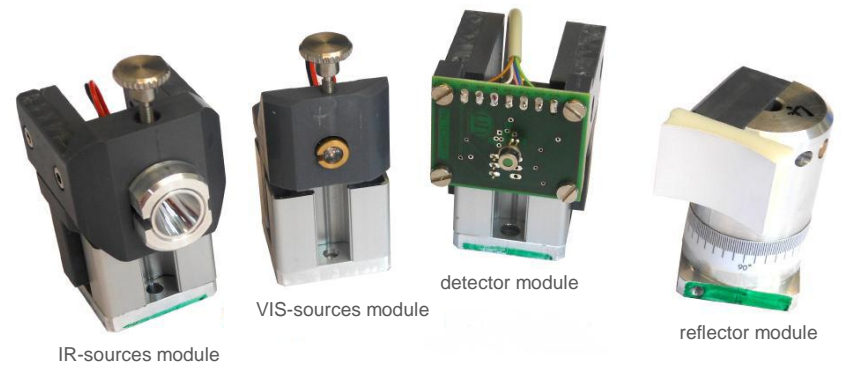

Figure 8. Modules of the IR-gas sensor construction kit.

With these modules the sample set-up was arranged with the specifications given by the optimized design as shown in Fig. 7. The fixation of the modules on the base plate was realized magnetically: magnets were embedded in the mounting structures of the optical modules as well as a magnetic material was used for the base plate. A photo of the set-up is presented in Fig. 9 where a plot of the configuration is arranged directly on the base plate for visualization of the beam path as well as for an adjustment aid.

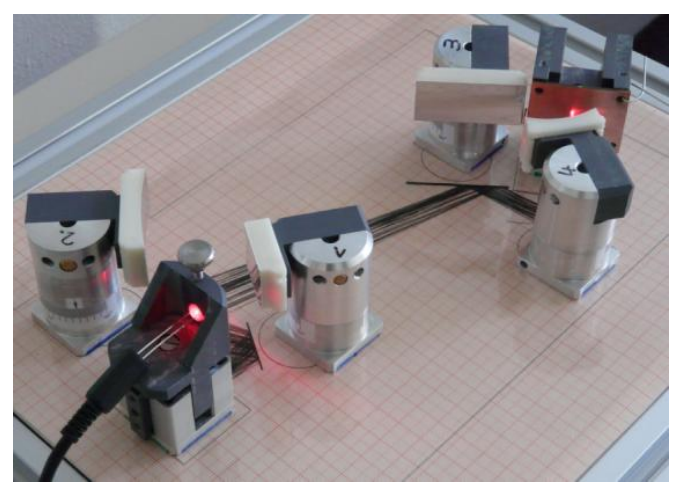

Figure 9. Set-up of the optical subsystem.

With the use of the VIS source module a tracing of the beam path was carried out to analyse and evaluate the beam forming by the mirrors. The spot was imaged at different positions in the beam path using a scale paper as screen. Fig. 10 shows the spot in three positions in between the mirrors two and three.
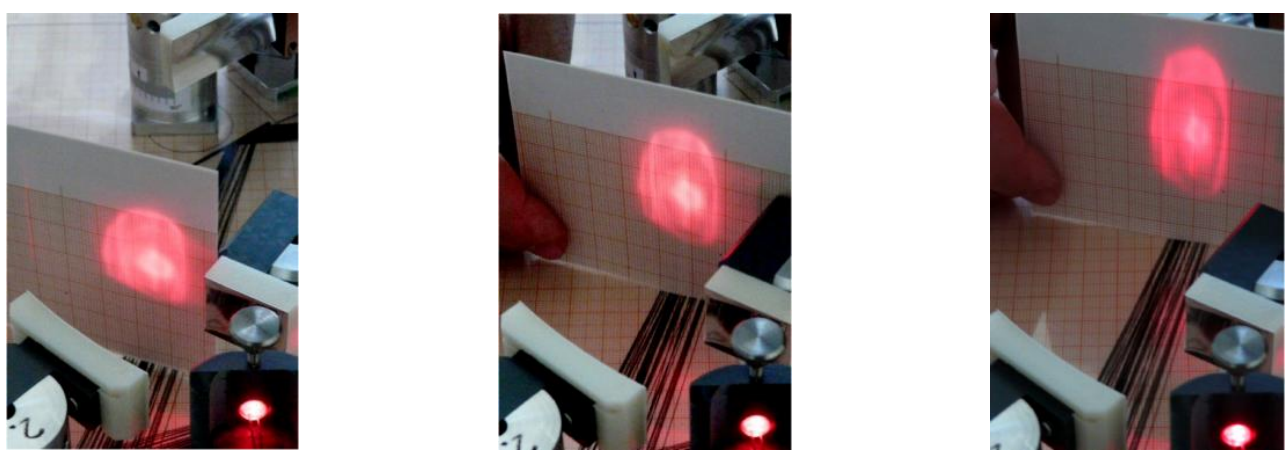

Figure 10. Beam spot at three positions in between the mirrors two and three.

The beam forming of the mirrors one and two can be observed clearly by looking at the spot immediately behind mirror two (Fig. 10, left). Passing the screen along the beam path towards mirror three an increase in vertical dimension of the spot is noticeable (Fig. 10, mid and right). While the collimation in the plane of the base plate carried out by the second mirror shows a pretty good behavior, beam divergence in the direction perpendicular to the base plate leads to a widening of the spot in this direction. Responsible for the insufficient beam forming perpendicular to the base plate is mirror 1 (see Fig. 7). It is assumed that the shape of mirror 1 does not meet the calculated shape from the optical simulation due to limitations of the additive manufacturing process.

Figure 11 shows a comparison of the imaged spot at the position of the detector with the simulated irradiance distribution at this position. Qualitatively considered the simulated irradiance resembles the imaged one in size and distribution. A quantitative assessment is currently still to be done. 

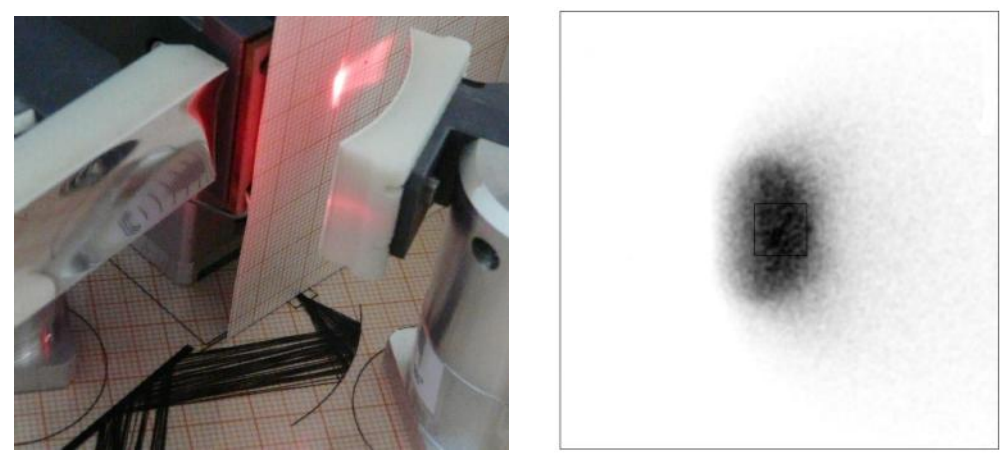

Figure 11. Comparison of the imaged beam spot at the target with the simulated irradiance distribution.

\section{CONCLUSIONS}

We have shown an approach to a cost-efficient modularly built non-dispersive IR-gas sensor based on a construction kit. The modules of the construction kit are interchangeable and freely fixable on a base plate to offer the opportunity to tune the NDIR to measuring different gasses with different absorption spectra with a specified sensitivity. This tuning can be done by choosing an adequate source and detector pair and the proper configuration of reflective elements in combination with a flexible analysis cell, respectively. Furthermore the developed approach is very well suited to be used in teaching. Together with students a construction kit on the basis of an optical free space system was developed and partly implemented to be further used as a teaching and training aid for bachelor and master students at our institute. We have shown the optimized design of a sample set-up using the components source, detector, and reflectors. The simulations of the reflective elements as well as of the optical subsystem was verified by means of a qualitative comparison to measurement results based on a realized sample set-up. This $L E G O \circledR$-like construction kit has the potential for rapid testing of a class of optical designs and as a teaching aid in student education.

The presented work proves the functioning of the approach in principle but further work has to be done to assess this quantitatively. To enable a quantitative evaluation of this approach an appropriate reconfigurable analysis cell has to be constructed together with the gas feed. With this additional components functioning and sensitivity of the sample set-up of the IR gas sensor can be evaluated quantitatively.

\section{REFERENCES}

[1] Sieber, I., Suphan, K.-H. "Model-based optimization of an infrared gas sensor," Proceeding of IASTED 62479, 334-338 (2003)

[2] Günzler, H., Heise, H. „IR-Spektroskopie“, S.3, VCH, Weinheim (1996).

[3] Gottwald, W, Wachter, G. „IR-Spektroskopie für Anwender“, S. 59, Wiley-VCH, Weinheim (1997).

[4] Sieber, I., Eggert, H., Suphan, K.-H., Nüssen, O. "Optical modeling of the analytical chamber of an IR gas sensor," Proc. SPIE 4408, 272-282 (2001).

[5] Sieber, I., Gengenbach, U. „Design and optimization of optical gas sensor systems”, in: Chemical Sensors-Simulation and Modelling, Momentum Press, New York, 405-453 (2013).

[6] http://www.laser2000.de/out/media/HWK-IR-50-Series.pdf (25.3.2015).

[7] http://finder.micro-hybrid.de/uploads/tx in2productfinder/MH_TS1x200B-B-D2.4.pdf (25.3.2015). 